Bangladesh J Med Microbiol 2020; 14 (1): 1-2

Bangladesh Society of Medical Microbiologists

\title{
Editorial
}

\section{Make your intention \\ Infection prevention}

\section{Professor Muhammad Akram Hossain ${ }^{1}$}

${ }^{1}$ Department of Microbiology, Imperial Hospital Limited, Pahartali, Chattogram, Bangladesh.

Healthcare associated infection represents a major public health concern worldwide in terms of mortality, costs and increased length of stay in healthcare settings. It is estimated that 1 in every 10 patients is affected by healthcare-associated infection worldwide. Out of every 100 patients, 7 in developed and 15 in developing countries will get at least one healthcare-associated infection ${ }^{1}$. In high-income countries, up to $30 \%$ of patients are affected by at least one healthcare-associated infection in intensive care units; in developing countries, the frequency is 2 to 3 times higher².

In 2018, a point prevalence survey conducted in hospitals in the USA estimated that $3.2 \%$ of patients had one or more HCAIs $^{3}$. In 2015, HCAI prevalence of 9\% was estimated in South East Asia ${ }^{4}$. The World Health Organization (WHO) observed that HCAI prevalence was highest in low-/middle-income countries, ranging from $5.7 \%$ to $19.1 \%^{2}$. Four types of infection accounts for more than $80 \%$ of all healthcare associated infections: respiratory tract infection, surgical-site infection, urinary tract infection and blood stream infection ${ }^{5}$. In Bangladesh there are only few studies conducted so far on infection control in hospital settings. One study in surgical ward of Dhaka Medical College revealed $30 \%$ of the study patient had suffered from nosocomial infection. Among them, $38.7 \%$ was wound infection, $26.6 \%$ urinary tract infection, $19.2 \%$ acute respiratory tract infection, and acute gastrointestinal infection was $12.5 \%$. Another significant finding of this study was that there was a strong positive association between the frequency of nosocomial infection and increasing numbers of visitors per patient per day ${ }^{6}$.

Health care-associated infections (HAIs) represent a major burden for health care system delivery and patient safety. However, evidence has clearly demonstrated that

Correspondence:

Professor Muhammad Akram Hossain

Senior Consultant, Department of Microbiology

Imperial Hospital Limited, Pahartali, Chattogram

Phone: 09612247247

Email: akram.prof@gmail.com effective implementation of evidence-based infection prevention and control (IPC) interventions can lead to substantial reductions-between $35 \%$ and $55 \%$ in $\mathrm{HAIs}^{5}$. The financial benefit of using these prevention practices is estimated worldwide to be $\$ 25$ billion to $\$ 31.5$ billion in medical cost savings. The emergence of the coronavirus disease-2019 (COVID-19) has again shown the importance of effective IPC implementation to prevent and control infectious disease outbreaks in health care ${ }^{7}$. Though in times of COVID pandemic, infection prevention and control (IPC) practices has increased manifolds. Unfortunately, an effective and robust infection control program is lacking in most of the healthcare facilities in Bangladesh.

World Health Organization (WHO) decided to prioritize the development of evidence-based recommendations on the essential elements ("core components") of IPC programs at the national and facility level which includes

1. IPC program

2. IPC guidelines

3. Education and training

4. HAI surveillance

5. Multimodal strategy

6. Monitoring/audit and feedback

7. Workload, staffing, and bed occupancy

8. Built environment, materials, and equipment for IPC at the facility level ${ }^{8}$.

These IPC core components offer an actionable structure for benchmarking healthcare systems and for supporting countries to work towards the goal of quality universal health coverage and health security for all.

Implementing infection control measures such as standard precautions like hand hygiene, personal protective equipment, environmental disinfection, equipment disinfection/ sterilization, etc., and transmission-based precautions can prevent the spread of microorganisms in health care 
settings. Microbiologist, clinicians and health administrators need to work together to ensure that each hospital health complex or clinic, irrespective of its size has an infection control program. In the long term, we need to ensure that infection control becomes a way of life for all health care workers. For this it is important that we incorporate the teaching of IPC principles in undergraduate and post graduate medical, nursing and allied science curriculum.

HAIs are a major source of budget drain on the hospital and patients. Limited resources represent the main challenge for governments in developing countries like Bangladesh. Therefore, improvement of current systems in terms of policy, practice, and process can serve more quality service in existing health care facilities which do not need much extra cost. Such understanding begins with awareness. To help in this fight against infection, a movement initiated by IPC alliance BD, a group of enthusiastic medical microbiologists for building awareness and supporting the government of Bangladesh with comprehensive training on infection prevention and control. The Directorate General of Health Services (DGHS), with the assistance of IPC alliance BD, trained 1000 physicians and nurses from upazilla health complex under eight division. A recent study from Bangladesh shows, although healthcare workers were formally trained on IPC, unsafe practices for injection, improper use of personal protective equipment, low level of adherence to hand hygiene and cough etiquette were frequent ${ }^{9}$. The study revealed that monitoring, audit and feedback of IPC measures is essential.

Effective IPC requires constant action at all health system levels, including policymakers, facility managers, health workers, and patients who access health services. This year's International Infection Control Week theme is "Make Your Intention Infection Prevention" with an aim to highlight the science behind infection prevention and inspiring the next generation of Infection preventionists to join the fight. We need to develop Hospital Infection Society in Bangladesh with an objective to disseminate information and recent updates on various aspects of healthcare associated Infections and their prevention. Also, it is needed to provide a common platform for personnel active in the field of prevention of healthcare associated infections, such as, Microbiologists, Administrators, Clinicians, etc.

\section{References}

1. Report on the Burden of Endemic Health Care Associated Infection Worldwide: World Health Organization: WHO press; 2011 May 5.p.1-34.

2. Allegranzi B, Nejad SB, Combescure C, Graafmans W, Attar H, Donaldson L, et al. Burden of endemic health-care-associated infection in developing countries: systematic review and meta-analysis. The Lancet. 2011 Jan 15;377(9761):228-41.

3. Magill SS, O'Leary E, Janelle SJ, Thompson DL, Dumyati G, Nadle J, et al. Changes in prevalence of health care-associated infections in US hospitals. New England Journal of Medicine. 2018 Nov 1;379(18):1732-44.

4. Ling ML, Apisarnthanarak A, Madriaga G. The burden of healthcare-associated infections in Southeast Asia: a systematic literature review and meta-analysis. Clinical Infectious Diseases. 2015 Jun 1;60(11):1690-9.

5. World alliance for patient safety; Global patient safety challenge: 2005-2006. World Health Organization; 2006 [Cited 2021 Nov 12]. Available at: https://apps.who.int/iris/handle/10665/43358.

6. Faruquzzaman. Positive associations of nosocomial infections in surgical ward with etiological clinical factors. Bratislava Medical Journal. 2011 Jan $1 ; 112(5): 273$

7. Bong CL, Brasher C, Chikumba E, McDougall R, Mellin-Olsen J, Enright A. The COVID-19 pandemic: effects on low-and middle-income countries. Anesthesia and analgesia. 2020 Apr 20.

8. Storr J, Twyman A, Zingg W, Damani N, Kilpatrick C, Reilly J, et al. Core components for effective infection prevention and control programmes: new WHO evidence-based recommendations. Antimicrobial Resistance \& Infection Control. 2017 Dec;6(1):1-8.

9. Alam M, Islam M, Khan M, Hamid S, Islam M, Laz S, et al. Current Status of Infection Control Practice in Surgical Settings of Leading Academic and Private Service Hospitals in Dhaka. Bangladesh. International Journal of Infectious Diseases and Therapy. 2021;6(3):97-115. 